\title{
Congenital Alveolar Proteinosis, A Straight Forward Diagnosis But Often Missed: A Case Report
}

\author{
Authors \\ Showkat Hussain Tali (MD, DNB) ${ }^{1}$, Nandkishore Shrikishanji Kabra (MD, DM) ${ }^{2}$, \\ Shagufta Yousuf (MD, PGDMCH) ${ }^{3}$, Javed Ahmed(MD) ${ }^{4}$, Phalgoni Padhi (MD, DNB) ${ }^{5}$, \\ Rashid Merchant (MD) ${ }^{6}$, Swarup Kumar Dash (MD, DNB) ${ }^{7}$ \\ ${ }^{1}$ Assistant Professor, ${ }^{2}$ Director, ${ }^{3}$ Assistant Professor, ${ }^{4,6}$ Consultant, ${ }^{5}$ Senior Resident \\ ${ }^{1}$ Department of Pediatics, AIMSR, Bathinda Punjab, India \\ ${ }^{2,4,5,6,7}$ Department of Neonatology, Surya Children's Hospital Mumbai, India Email: drnskabra@gmail.com \\ ${ }^{3}$ Department of OBG, AIMSR, Bathinda, Punjab, India; Email: drshaguftashifa@gmail.com \\ Corresponding Author \\ Tali Showkat Hussain \\ Assistant Professor Pediatrics, AIMSR, Bathinda, Punjab, India \\ Email: drshowkatshifa@gmail.com, Pin code: 151001.Ph. 7006209304, 9167641630
}

\begin{abstract}
We report an infant who presented with respiratory distress soon after birth. After exhaustive workup including histopathalogy and electronmicroscopy of the excision biopsy specimen of the lung, infant was finally diagnosed to have congenital alveolar proteinosis.
\end{abstract}

Keywords: Neonate, Congenital, Alveolar proteinoses, Respiratory distress.

\section{Introduction}

PAP was first described in $1958 .{ }^{(1)}$ PAP is an extremely rare cause of respiratory failure in the pediatric age group. PAP is characterized by intraalveolar accumulation of surfactant, namely lipid and proteinaceous material that is periodic acidSchiff (PAS) positive when visualized on light microscopy. ${ }^{(2,3)}$ The disease is not associated with inflammation, and lung architecture is typically preserved. Impairment of surfactant clearance as a result of inhibition of the action of GM-CSF may underlie many acquired cases, whereas congenital disease is most commonly attributable to mutations in surfactant protein genes but may also be caused by GM-CSF receptor defects ${ }^{(4,5)}$.Three clinically distinct forms of PAP have been described: autoimmune (primary or idiopathic), secondary, and genetic (congenital) ${ }^{(6,7,8)}$ Genetic PAP is seen more commonly in children, while adult forms are usually autoimmune. (2,6,9,10) $^{-}$

\section{Case Report}

This near term, $36+4$ weeks, $2.8 \mathrm{Kg}$ birth weight, AGA, non consangeneous product, male infant was admitted to Surya child Care neonatal Intensive Care Unit (SCH-NICU) at day 23 of life with respiratory distress since birth. Infant was born to a 32 years G2P1L1 mother. Antenatal period was 
uneventful. It was a LSCS delivery for non reassuring fetal status. Baby was vigorous at birth and resuscitation was not required. However, infant was noticed to have respiratory distress soon after, for which infant was admitted to a NICU at Indore, India. At admission infant was given one dose of surfactant for suspected Respiratory distress syndrome. Infant was ventilated up to day 8 of life, given one more dose of surfactant at day 8 of life, extubated and put on oxygen supplementation through nasal prongs. Infant was reintubated at day 11th of life for increasing respiratory distress and inability to maintain blood gases and $\mathrm{PH}$ within normal limits. Chest X-rays showed persistent bilateral haziness. Infant was given multiple antibiotics with suspicion of congenital pneumonia. However there were no significant antenatal risk factors for sepsis and infant's sepsis work-up was unremarkable. CT chest showed patchy areas of air space consolidation bilaterally. As respiratory distress was persistent, infant was transferred at 23 days of life to Surya Child Care NICU, Mumbai for further care.

At admission infant's general physical examination revealed features of respiratory distress in the form of tachypnea and intercostal and subcostal retractions. Infant's initial arterial blood gas analysis was s/o severe chronic respiratory acidosis. Infant was put on ventilatory support. IV antibiotics were started following performing work-up for sepsis. RT feeds were started as full feeds at admission and were tolerated satisfactorily. Differential diagnoses considered, included; (1) Unresolving pneumonia; (2) Congenital heart disease (TAPVC); (3) GERD; (4) H type Tracheoesophageal fistula; (5) Immunodeficiency; (6) Cystic fibrosis; (7) Alpha 1 antitrypsin deficiency; (8) Congenital Pulmonary alveolar proteinosis; (9) Congenital Lymphangeictasia (10) Primary Ciliary Dyskinesia (11) CMV pneumonitis. Respiratory distress persisted throughout the admission. Chest X-rays performed periodically showed persistent hazziness. Respiratory assistance in the form of Ventilatory support, CPAP support and HHHFNC supports were required throughout the admission.
Periodic sepsis screens were negative. Blood culture collected at admission and during the course of admission remained sterile. CSF study was unremarkable. Tracheal secretions collected periodically for culture were suggestive of colonization. CMV antibody titers were nonreactive and Infant's CMV DNA-PCR was also negative. Work-up for immunodeficiency including flow cytometric lymphocyte subset analysis was unremarkable. 2D ECHO of the heart performed on day 23 of life did not reveal any structural anomaly. Cranial ultrasound and USG abdomen and pelvis were normal. Milk scan performed revealed presence of high grade (IV) gastro esophageal reflex.. Along with general antireflex measures fundoplication was performed. Repeat milk scan performed showed no evidence of GER. Infant's HRCT performed revealed diffuse ground glass opacities bilaterally sparing left lower lobe, suggestive of alveolar edema of uncertain cause with normal tracheo-bronchial tree. Work-up for cystic fibrosis (delta 508 mutation) and alpha 1 antitrypsin deficiency were unremarkable. Electron microscopy of nasal scraping for Cilia morphology was unremarkable. Bronchoalveolar lavage revealed lipid laden macrophages but little PAS positive staining. Histopathological examination of the excisional lung biopsy revealed PAS positive material in alveolar spaces with preserved alveolar architecture. Electron microscopy examination was suggestive of congenital alveolar proteinosis. For financial constraints further evaluation could not be performed. Despite giving optimal supportive care, performing partial broncho-alveolar lavage (once), administering IVIG, Methylpredinosolone, and granulocyte colony stimulating factor, no significant response was observed. On request, infant was transferred back to Indore, were he died peacefully in an NICU after 3 days of transfer.

\section{Discussion}

Our patient was a near term baby with respiratory distress since birth, requiring respiratory support in the form of mechanical ventilation and continuous positive airway pressure. As is typical of congenital 
alveolar proteinosis ${ }^{(11)}$, respiratory distress was persistent since birth and throughout the admission. Although the disease is mostly transmitted in autosomal recessive manner, ${ }^{(12)}$ there was no history of consanguinity or positive family history. So absence of consanguinity or a positive family history does not rule out possibility of congenital alveolar proteinosis.

In neonates with congenital variety, the mortality rate associated with conventional therapy is $100 \%$ ${ }^{(13)}$. This was true for our patient as well as despite doing all possible measures, patient could not be salvaged. There is no specific treatment of PAP. Some kind of respiratory support is often necessary in congenital PAP. Repeated whole lung lavage is the mainstay of treatment for PAP in older children but in neonates it is usually difficult to perform and often ineffective. The aim is to eliminate the material in distal air spaces and restore the permeability of the alveolar-capillary barrier ${ }^{(14)}$. The use of whole-lung lavage is less well established in young infants and newborns mainly because of the technical difficulties associated with use of large endotracheal tube ${ }^{(15)}$. In coordination with a pediatric surgeon and a pediatric ENT surgeon we attempted alveolar lavage both for therapeutic and diagnostic purpose. There was no significant relief in symptoms and the material obtained from lavage was too small to be helpful for a diagnostic purpose.

The use of surfactant has not been of benefit. Intravenous immunoglobulin (IVIG) and GM-CSF therapy do not have a role in congenital form of disease although in adult studies they have shown benefit ${ }^{(14,16,17)}$. Our patient had already received two doses of surfactant before transfer for suspected RDS but there was no significant benefit. Infants suffering from congenital alveolar proteinosis are often administered surfactant for suspected respiratory distress syndrome. When there is no response to surfactant, congenital alveolar proteinoses becomes a strong possibility. At the request of parents IVIG, methyl predinosolone and GM-CSF were used but of no avail. Other options include extra-corporeal membrane oxygenation and lung transplantation ${ }^{(14)}$. Although Lung transplantation is known to improve survival, this option could not be considered in our patient because of financial constraints. Because congenital PAP is a single-gene defect, it may be a candidate disease for gene therapy ${ }^{(14)}$.

\section{Conclusion}

Congenital pulmonary alveolar proteinoses is often not considered for diagnostic evaluation in persistent respiratory distress since birth in developing countrie even if the diagnosis is straight farword. The possible reasons being the rarity of the condition, costly and difficult workup and non availability of definitive and permanent cure. We strongly recommend that it should be always considered in differential diagnosis and worked up whenever possible to avoid unnecessary extensive diagnostic and therapeutic interventions and to offer timely counseling to the family.

\section{Acknowledgement}

We are thankful to dr Rahul, dr Bhargavi, dr Vipul dr Dhawal, and dr Nilesh for their help and support during the study.

\section{Bibliography}

1. Rosen SH, Castleman B, Liebow AA. Pulmonary alveolar proteinosis. N Engl J Med 1958; 258: 1123-43.

2. Trapnell BC, Whitsett JA, Nakata K. Pulmonary alveolar proteinosis. N Engl J Med 2003; 349(26):2527-39.

3. [Guideline] Leth S, Bendstrup E, Vestergaard $\mathrm{H}$ and Hilberg O. Autoimmune pulmonary alveolar proteinosis: treatment options in year 2013. Respirology 2013; 18:82-91.

4. Wasserman K, Mason GR. Pulmonary alveolar proteinosis. In: Murray JF, Nadel JA, editors. Textbook of Respiratory Medicine, 5th ed. Philadelphia: Saunders 1994;1933-46

5. Teja K, Cooper PH, Squires JE, Schatterly PT. Pulmonary alveolar proteinosis in four siblings. N Engl J Med 1981; 305: 1390-92. 
6. Patel SM, Sekuguchi H, Reynold J and Krowks M. Pulmonary alveolar proteinosis. Canadian Respiratory Journal 2012; 19:243-45.

7. Raj D, Bhutia TD, Mathur S, Kabra SK, Lodha R. Pulmonary alveolar proteinosis secondary to Pneumocystis jiroveci infection in an infant with common variable immunodeficiency. Indian

Pediatr. 2014;81:929-31

8. Hammami S, Harrathi K, Lajmi K, Hadded $\mathrm{S}$, Ben Meriem C, Guédiche MN. Congenital pulmonary alveolar proteinosis. Case Rep Pediatr 2013:764216. doi: 10.1155/2013/764216

9. Samuels MP, Warner JO. Pulmonary alveolar lipoproteinosis complicating juvenile dermatomyositis. Thorax. Nov 1988; 43(11):939-40.

10. deMello DE, Nogee LM, Heyman S, et al. Molecular and phenotypic variability in the congenital alveolar proteinosis syndrome associated with inherited surfactant protein B deficiency. J Pediatr 1994; 125(1):43-50.

11. Blaivas AJ. Pulmonary alveolar proteinosis. MedlinePlus. May 21, 2009;

12. Kniffin, CL. Surfactant metabolism dysfunction, pulmonary 1. OMIM. April 24, 2007;

13. Parto K, Kallojoki M, Aho H, Simell O. Pulmonary alveolar proteinosis and glomerulonephritis in lysinuric protein intolerance, case report and autopsy finding of four paediatric patients. Hum Path 1994; 25: 400-7.

14. deBlic J. Pulmonary alveolar proteinosis in children. Pediatr Resp Rev 2004; 5; 316-22.

15. Ramirez RJ, Campbell GD. Pulmonary alveolar proteinosis: endobronchial treatment. Ann Intern Med 1965; 63: 429-41.

16. Shah PL, Hansell D, Lawson PR. Pulmonary alveolar proteinosis: clinical aspects and current concepts on pathogenesis. Thorax 2000; 55: 67-77.
17. Trapnell BC, Whitsett JA, Nakata K. Pulmonary alveolar proteinosis. N Engl J Med 2003; 349: 2527-39. 